University of Nebraska - Lincoln

DigitalCommons@University of Nebraska - Lincoln

$5-15-1992$

\title{
Evidence for the formation of metallic mercury overlayers on $\mathrm{Si}(111)$
}

Dongqi Li

Syracuse University

Jiandi Zhang

Syracuse University, jiandiz@lsu.edu

Sunwoo Lee

Syracuse University

Peter A. Dowben

University of Nebraska-Lincoln and Syracuse University, pdowben@unl.edu

Follow this and additional works at: https://digitalcommons.unl.edu/physicsdowben

Part of the Physics Commons

Li, Dongqi; Zhang, Jiandi; Lee, Sunwoo; and Dowben, Peter A., "Evidence for the formation of metallic mercury overlayers on Si(111)" (1992). Peter Dowben Publications. 60.

https://digitalcommons.unl.edu/physicsdowben/60

This Article is brought to you for free and open access by the Research Papers in Physics and Astronomy at DigitalCommons@University of Nebraska - Lincoln. It has been accepted for inclusion in Peter Dowben Publications by an authorized administrator of DigitalCommons@University of Nebraska - Lincoln. 


\title{
Evidence for the formation of metallic mercury overlayers on $\mathrm{Si}(111)$
}

\author{
Dongqi Li, Jiandi Zhang, Sunwoo Lee, and P. A. Dowben* \\ Department of Physics, Syracuse University, Syracuse, New York 13244-1130 \\ (Received 23 September 1991; revised manuscript received 2 December 1991)
}

\begin{abstract}
A strongly chemisorbed $\mathrm{Hg}$ species is observed following the adsorption of $\mathrm{Hg}$ on $\mathrm{Si}(111)$ at $110 \mathrm{~K}$. This strongly chemisorbed $\mathrm{Hg}$ species and subsequently weakly chemisorbed $\mathrm{Hg}$ species are observed to be metallic in the photoemission final state as determined by resonant photoemission and the reduction of photovoltaic charging. We postulate that the strongly chemisorbed $\mathrm{Hg}$ species on $\mathrm{Si}(111)$ is more metallic at dilute coverages than $\mathrm{Hg}$ adsorbed on $\mathrm{Cu}(100)$.
\end{abstract}

\section{INTRODUCTION}

The fractional occupation of the $\mathrm{Hg} 6 s$ level, or the formation of highly coordinated mercury overlayers, is believed to result in the formation of the metallic mercury overlayer. ${ }^{1}$ Some mercury overlayers on the surfaces of metals [such as $\mathrm{Cu}(100)$ ] are seen to be nonmetallic in the photoemission final state because of the unusual electronic properties of the group-II metals. ${ }^{1}$

Recently, investigations of alkali-metal adsorption on $\mathrm{Si}(100)$ (Refs. 2-4) provided evidence for the formation of a metallic surface at submonolayer coverages of alkali metals. With any appreciable charge transfer from $\mathbf{H g}$ adatoms to silicon, $\mathrm{Hg}$ overlayers would be expected to exhibit similar behavior. In other words, strong chemisorption bonds between $\mathrm{Hg}$ and $\mathrm{Si}(111)$ may result in a more metallic $\mathrm{Hg}$ overlayer on $\mathrm{Si}(111)$ than is observed with submonolayer $\mathrm{Hg}$ overlayers on $\mathrm{Cu}(100)$. These metallic overlayers are expected to be similar in character to higher-coverage $\mathrm{Hg}$ overlayers on metallic substrates such as $\mathrm{Cu}(100)$.

While the investigation, using photoemission, of the metal-silicon (111) interface has been given considerable attention, ${ }^{6-13}$ no study of the $\mathrm{Hg}$-Si(111) interface has been, as yet, reported. Nonetheless, by comparing results for $\mathrm{Hg}$ on $\mathrm{Si}(111)$ with results for $\mathrm{Hg}$ adsorbed on other substrates, we are able to demonstrate that the $5 d \rightarrow \epsilon f$ resonant photoemission excitation is very sensitive to changes in electron screening lengths in ultrathin metal overlayers. This sensitivity is greater for changes in screening lengths for ultrathin films than is the case for thicker films. Simple theoretical models support the postulate that the resonant photoemission intensities become weaker with increasing electron itinerancy. ${ }^{1,5}$ For twodimensional systems the screening effect is much weaker than for three-dimensional systems.

\section{EXPERIMENTAL DETAILS}

The photoemission experiments were undertaken in an angle-resolved photoemission system equipped with a hemispherical electron-energy analyzer and low-energy electron diffraction (LEED) described in detail elsewhere. ${ }^{14}$ All the photoemission spectra were taken with the electrons collected normal to the surface with a light incidence angle of $45^{\circ}$ off the surface normal. The photon energy throughout this work was $55 \mathrm{eV}$. The light source was the 1-GeV ring at the Synchrotron Radiation Center, dispersed by a $3-\mathrm{m}$ toriodal grating monochromator. Binding energies reported throughout these studies are with respect to the metallic mercury Fermi level. The low-energy-electron-diffraction studies were undertaken in a reverse-view retarding-field analyzer.

The partial photoemission cross sections or constant initial-state (CIS) spectra were carried out as described elsewhere. ${ }^{1,5,14}$ The kinetic and photon energies were changed together, so as to determine the relative photoemission intensity of a given initial state (say, for example, the $\mathrm{Hg} 5 d_{5 / 2}$ feature). The CIS spectra were normalized for photon flux through the monochromator using the current from a gold diode at the exit of the monochromator.

The samples were polished $n$-type $\mathrm{Si}(111)$ doped to about $7 \times 10^{14} \mathrm{~cm}^{-3}$, though there is considerable evidence that our surface-preparation procedure leaves the surface $p$ type. The samples were prepared in situ. ${ }^{15}$ Lengthy annealing of the sample was also undertaken at $900^{\circ} \mathrm{C}$ following flashing of the crystal to $1250^{\circ} \mathrm{C}$. This methodology was found to give a $7 \times 7$ reconstruction of the $\mathrm{Si}(111)$ surface, as well as clear indication, in photoemission, of all the $\mathrm{Si}(111)$ surface states, which have been characterized in detail. ${ }^{16}$ The sample temperature was determine using a Chromel-Alumel thermocouple, an optical pyrometer, and thermoresistivities. All pressures reported in this work are uncorrected for the ionizationgauge cross section of mercury.

\section{RESULTS}

In order to minimize the mercury diffusion into the silicon substrate and reduce the mobility of the silicon carriers, the photoemission studies were carried out for mercury adsorption on $\mathrm{Si}(111)$ at $110 \mathrm{~K}$, as shown in Fig. 1 . As with all studies on semiconductors, defining the Fermi level presents some difficulties. By adsorbing very thick films of $\mathrm{Hg}$ on $\mathrm{Si}(111)$ at $110 \mathrm{~K}$, the silicon features can be completely obscured (as indicate in Fig. 2) and a Fermi level defined by metallic mercury can be identified (as seen in Fig. 1).

Because of the low temperature $(110 \mathrm{~K})$, photovoltage 
effects in photoemission dramatically effect the position of the valence-band edge, ${ }^{17-27}$ particularly at the surface. The adsorption of mercury has a dramatic effect on this temperature-dependent surface photovoltage (as seen in Fig. 3). The valence-band edge, determined from the photoemission spectra (energy-distribution curves), rapidly approaches the metallic mercury Fermi level. The magnitude of the shift $(0.55 \pm 0.05 \mathrm{eV})$ is consistent with results obtained for clean $p$-type silicon with the $7 \times 7$ reconstruction. ${ }^{19}$ This band-bending effect diminishes with increasing mercury coverage, as seen in Figs. 1 and 3. This effect has been noted by Alonso and coworkers, ${ }^{17}$ Stiles et al., ${ }^{25,28}$ and Wadill and co-workers ${ }^{18}$ for other metal overlayers on semiconductor surfaces.

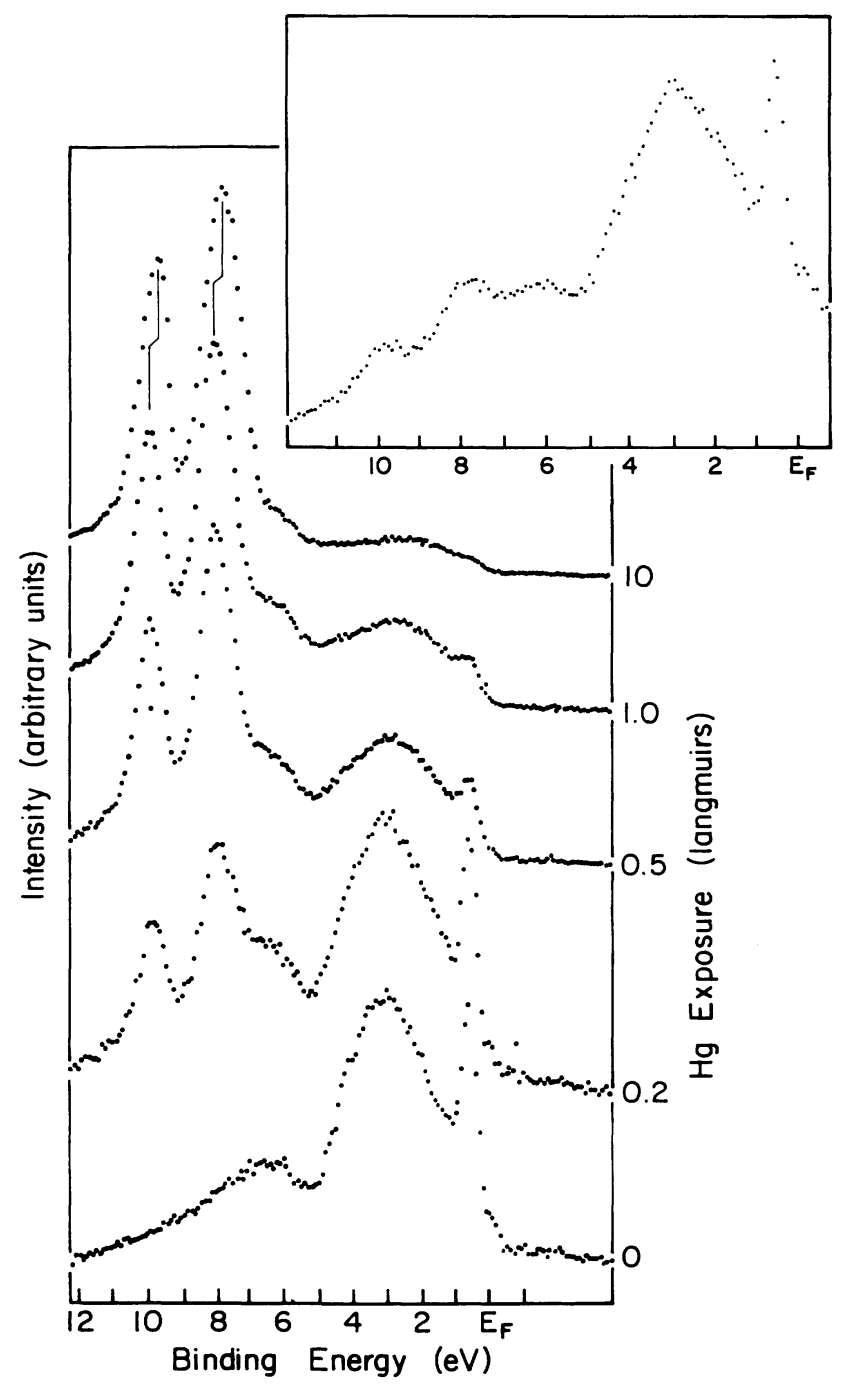

FIG. 1. Photoemission spectra of mercury adsorption on $\mathrm{Si}(111)$ at $110 \mathrm{~K}$. The photon energy is $53 \mathrm{eV}$ and the light incidence angle is $45^{\circ}(s+p$ polarized light). All photoelectrons were collected normal to the surface. The inset shows the surface following annealing to $1250 \mathrm{~K}$. All binding energies are with respect to the mercury Fermi energy and do not account for surface photovoltage effects (if any).

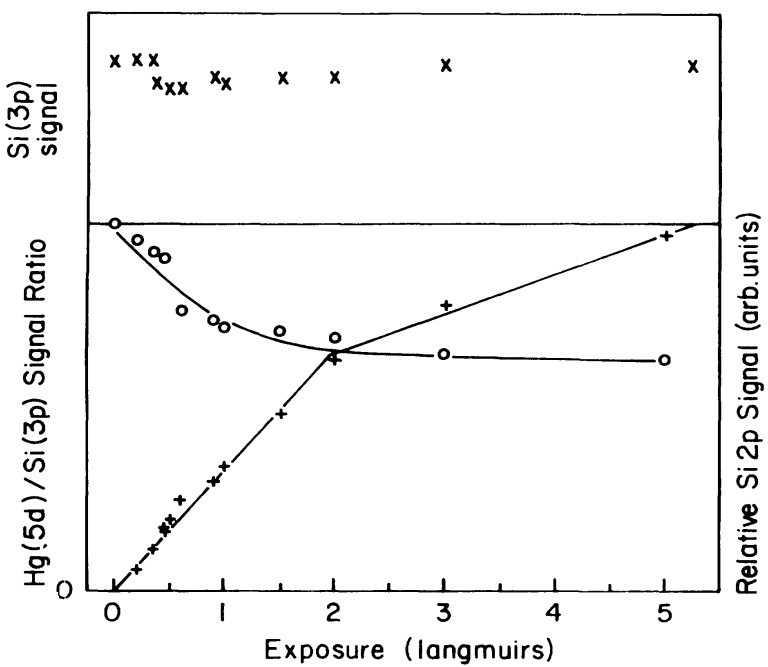

FIG. 2. Silicon photoemission $3 p(X)$ and $2 p(O)$ signals with initial $\mathrm{Hg}$ exposure to $\mathrm{Si}(111)$ at $110 \mathrm{~K}$. The $\mathrm{Hg}$-to-Si valence-band signal ratio $(+)$ is included for reference.

Two pronounced features are observed with increasing mercury exposure to $\mathrm{Si}(111)$ at $110 \mathrm{~K}$, as seen in Fig. 1 . With small exposures less than $1 \mathrm{~L}$ ( $\equiv 1 \times 10^{-6}$ Torr sec), these two features appear at $8.1 \pm 0.05$ and $10.0 \pm 0.05 \mathrm{eV}$ with respect to the metal Fermi level (binding energies of more than 8.4 and $10.3 \mathrm{eV}$ with respect to the apparent valence-band edge). With increasing coverage these features decrease in binding energy to $7.75 \pm 0.05$ and $9.65 \pm 0.05 \mathrm{eV}$, respectively, by $5 \mathrm{~L}$ or more $\mathrm{Hg}$ exposure, as seen in Fig. 1. From a comparison with mercury adsorbed on other surfaces, ${ }^{1,29-35}$ these two features can be identified as the spin-orbit-split $5 d$ levels of mercury.

Unlike $\mathrm{Hg}$ on some other substrates, ${ }^{31,34}$ there is no clear indication of a sharp (narrow half-width) $\mathrm{Hg} 6 \mathrm{~s}$ feature well away the Fermi level, at large $\mathrm{Hg}$ coverages. At low $\mathrm{Hg}$ coverages any sharp "atomiclike" $6 s$ feature is obscured by the silicon density of states. Rather, the $\mathrm{Hg}$

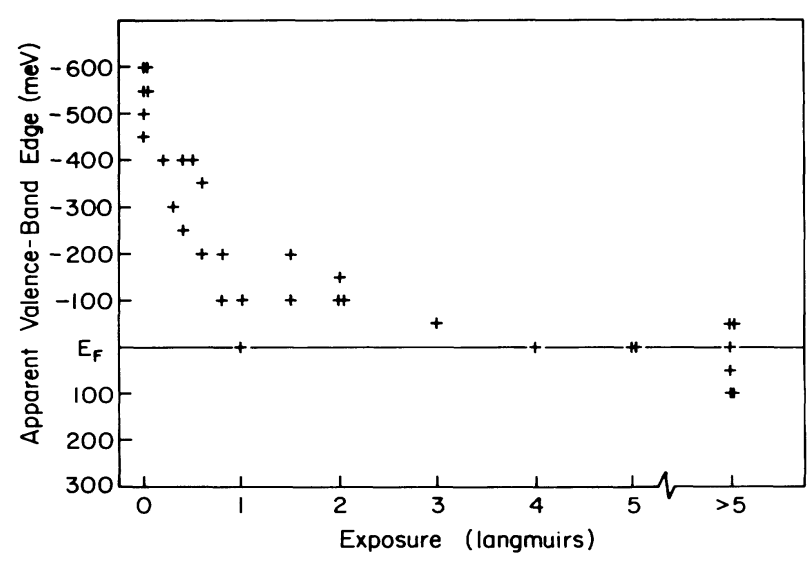

FIG. 3. Movement of the apparent valence-band edge with increasing mercury exposure to $\mathrm{Si}(111)$ at $110 \mathrm{~K}$. 
induces a broad photoemission intensity, as is seen for metallic Hg (Ref. 33) and for thick films [several monolayer films on $\operatorname{Ag}(100)$ (Ref. 30) and $\mathrm{Cu}(100)$ ]. ${ }^{1,34}$ This intensity replaces the $\mathrm{Si}(3 p)$ intensity (as suggested by Fig. 2 ), but the extent of this "replacement" is difficult to determine.

With annealing the greater portion of adsorbed $\mathrm{Hg}$ is seen to desorb by room temperature, but there exists a small amount of adsorbed mercury that remains tenaciously bound (at elevated temperatures $T>500 \mathrm{~K}$ ) to the surface and cannot be removed even with annealing to $1250 \mathrm{~K}$. This result is indicate by the presence of $\mathrm{Hg}$ $5 d$ features even after annealing $1250 \mathrm{~K}$, as seen in Fig. 1 . As discussed in detail elsewhere, ${ }^{36}$ the $\sqrt{3} \times \sqrt{3}$ structure adopted with $10-20 \mathrm{~L}$ exposure to $\mathrm{Si}(111)$ at $150 \mathrm{~K}$ is seen to persist with annealing to $1000 \mathrm{~K}$. Further repeated annealing to $1250 \mathrm{~K}$ does restore to surface $7 \times 7$ reconstruction associated with clean $\mathrm{Si}(111)$. The $1 \times 1$ LEED pattern observed following $38 \mathrm{~L}$ exposure to $\mathrm{Si}(111)$ at $150 \mathrm{~K}$ is seen to persist with annealing to 1250 K. The persistence of mercury adsorbed on $\mathrm{Si}(111)$ necessitated repeated changes of $\mathrm{Si}(111)$ substrates throughout this work.

The $\mathrm{Hg} 5 d_{5 / 2}$-feature envelope is often observed to be separated into two features. ${ }^{1,29-31,33-35}$ This half-width provides an indication of the degree of $\mathrm{Hg}$ adatom hybridization with adjacent atoms. It is therefore important to compare the $\mathrm{Hg} 5 d_{5 / 2}$-feature half-width with the corresponding half-width observed for $\mathrm{Hg}$ adsorbed on other surfaces, as seen in Fig. 4. While for $\mathrm{Hg}$ on $\mathrm{Cu}(100)$ the $5 d_{5 / 2}$-level half-width is seen to increase dramatically with coverage, as noted elsewhere, ${ }^{1,34}$ for $\mathrm{Hg}$ on $\mathrm{Si}(111)$, the increase in half-width is only about $0.25 \mathrm{eV}$, and at dilute coverages the $\mathrm{Hg} 5 d_{5 / 2}$ half-width is nearly double that observed for $\mathrm{Hg}$ on $\mathrm{Cu}(100)$.

As seen in Fig. 5, the $\mathrm{Hg} 5 d$ photoemission partial cross section varies little with coverage. The photoemission partial cross section exhibits a broad maximum peaked at about $59 \mathrm{eV}$ and is generally similar to that observed for mercury on other substrates, ${ }^{1,37-39}$ but peaked

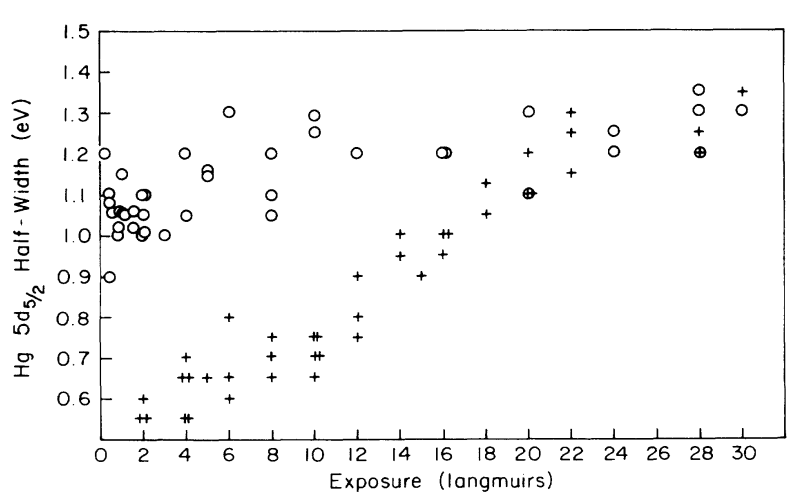

FIG. 4. $5 d_{5 / 2}$ full width at half maximum for $\mathrm{Hg}$ adsorbed on $\mathrm{Si}(111)$ at $110 \mathrm{~K}(O)$ and on $\mathrm{Cu}(100)$ at $200 \mathrm{~K}(+)$. This full width at half maximum includes all features within the $5 d_{5 / 2}$ envelope (including the splitoff $5 d$ state created as a result of band-structure formation).

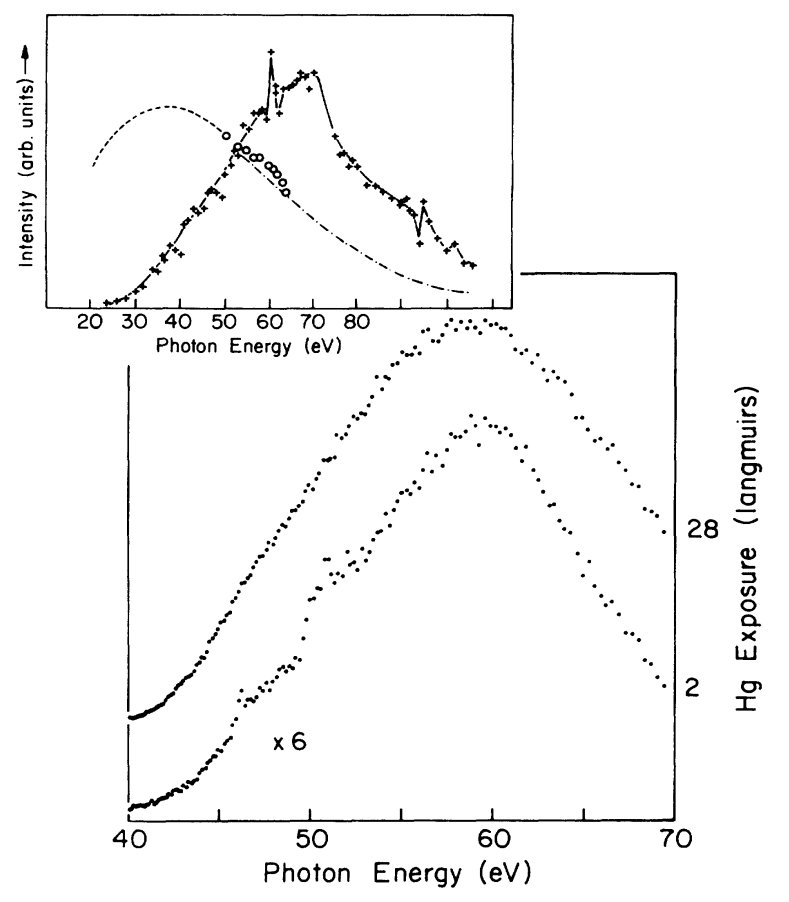

FIG. 5. $5 d_{5 / 2}$ constant initial-state photoemission spectra for $\mathrm{Hg}$ on $\mathrm{Si}(111)$ at $110 \mathrm{~K}$. The inset shows the spectra for gaseous $\mathrm{Hg}$ (Refs. 40-42), a disordered 5-ML film of $\mathrm{Hg}$ on $\mathrm{Ag}(100)$ at $30 \mathrm{~K}(\mathrm{O})$ taken from Ref. 38, and an ordered 5-ML film of $\mathrm{Hg}$ on $\mathrm{Ag}(100)$ at $90 \mathrm{~K}(+)$ taken from Refs. 37-39. The disordered $\mathrm{Hg}$ overlayer and gaseous $\mathrm{Hg}$ are representative of nonmetallic $\mathrm{Hg}$, while the ordered $\mathrm{Hg}$ overlayer on $\mathrm{Ag}(100)$ is an extremely metallic $\mathrm{Hg}$ overlayer.

at a photon energy far greater than observed for gaseous Hg. ${ }^{39-42}$

\section{DISCUSSION}

The persistence of adsorbed mercury on $\mathrm{Si}(111)$ to temperatures as high as $1250 \mathrm{~K}$ is indicative of the existence of an extremely strongly chemisorbed species. Under UHV conditions $\mathrm{Hg}$ adsorbed on $\mathrm{Ag}(100),{ }^{30,43}$ $\mathrm{Cu}(100),{ }^{43,44} \mathrm{Ni}(100),{ }^{45} \mathrm{Ni}(111),{ }^{46} \mathrm{~W}(100),{ }^{47}$ and $\mathrm{Fe}(100)$ (Ref. 48) is seen to desorb completely by $250-450 \mathrm{~K}$, much lower than is observed on $\mathrm{Si}(111)$. Thus, while mercury only weakly chemisorbs on transition metals, some mercury on $\mathrm{Si}(111)$ is strongly chemisorbed.

The photoemission and LEED results demonstrate ${ }^{36}$ that some mercury is strongly bound to the $\mathrm{Si}(111)$ surface. The mercury-induced $\sqrt{3} \times \sqrt{3}$ reconstruction of $\mathrm{Si}(111)$ has been noted with the adsorption of many other metals on $\mathrm{Si}(111)$ (Ref. 49) and is consistent with strong chemisorption. ${ }^{36}$ The half-width of the $\mathrm{Hg} 5 d_{5 / 2}$ feature provides a clear indication of the initial $\mathrm{Hg}$ adatoms strongly chemisorbed to $\mathrm{Si}(111)$. At very lower coverage the $\mathrm{Hg} 5 d_{5 / 2}$ envelope is very large compared with the photoemission half-width of this pair of features for $\mathrm{Hg}$ on other surfaces, for example, on $\mathrm{Cu}(100)$, at similar $\mathrm{Hg}$ exposures. On $\mathrm{Cu}(100)$ (Ref. 1) and W(100) (Ref. 31), the 
narrow $5 d_{5 / 2}$ half-width is indicative of the absence of two-dimensional band-structure formation or strong chemisorption with the substrate. As the mercury coverage increases on $\mathrm{Cu}(100)$ (Ref. 1) and $\mathrm{Ag}(100),{ }^{1,29}$ a twodimensional overlayer band structure is formed, resulting in an increase in the $5 d_{5 / 2}$ full width at half maximum, as shown in Fig. 4. Thus the very large $5 d_{5 / 2}$ mercury halfwidth, relative to the half-widths observed on $\mathrm{Cu}(100)$ (Fig. 4), is consistent with initial mercury adsorption leading to the formation of a strongly chemisorbed mercury species. The electronic structure of $\mathrm{Hg}$ adatoms, even at the most dilute coverages, is clearly different for $\mathrm{Hg}$ on $\mathrm{Si}(111)$ as compared with $\mathrm{Hg}$ on $\mathrm{Cu}(100)$.

Similarly, the larger $\mathrm{Hg} 5 d$ binding energies observed at low coverages are also indicative of bonding to the $\mathrm{Si}(111)$ and the formation of a strong chemisorption bond. $^{6}$ As the mercury coverage increases, the mercury becomes increasingly placed within a mercury lattice and the binding energies reflect this. The shift of the $\mathbf{H g} 5 d$ levels to smaller binding energy must also take into account the diminishing photovoltage with increasing coverage. The shift of the $\mathrm{Hg} 5 d$ binding energies to greater binding energies at low coverages is clearly opposite to the effect that the surface photovoltage effect has upon the photoemission feature (which is to decrease the binding energies as seen in Figs. 1 and 3).

While island growth of $\mathrm{Hg}$ on $\mathrm{Si}(111)$ at $100 \mathrm{~K}$ cannot

ㅁ square lattice - theory

$\Delta$ hexagonal lattice - theory

- square lattice - experiment

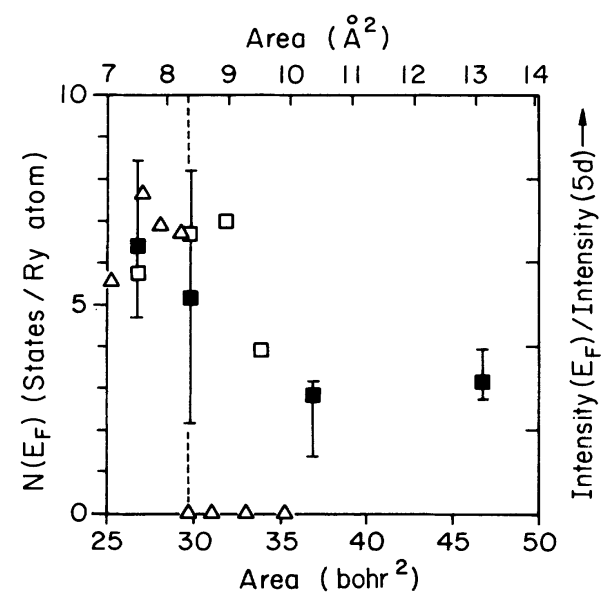

FIG. 6. Calculated densities of states at the Fermi energy as a function of atomic area for a square lattice ( $\square$ ) and a hexagonal lattice $(\triangle)$ taken from Ref. 69 . The relative intensities of the $\mathbf{H g}$-induced photoemission feature at the Fermi energy for a variety of $\mathrm{Hg}$ monolayers in a square lattice are shown ( $\square$ ) and are taken from Ref. 1. The dashed line indicates the predicted metal-insulator transition of $\mathrm{Hg}$ in a hexagonal lattice (Ref. 69) and is in agreement with the structural phase transition observed by Singh and Jones (Ref. 35). be excluded from our data, substantial clustering of $\mathrm{Hg}$ at the most dilute coverages of $\mathrm{Hg}$ on $\mathrm{Si}(111)$ is difficult to reconcile with our photoemission results (as seen in Fig. 1). Forming clusters sufficiently large so as to be metallic, ${ }^{50}$ for $\mathrm{Hg}$ exposures less than $5 \mathrm{~L}$ exposure, would result in substantial signal from the $\mathrm{Si}(111)$ substrate. The photoemission intensities are suggestive of layer-by-layer growth (as seen in Fig. 2), though by no means as conclusive as layer-by-layer growth noted for other $\mathrm{Hg}$ overlayer. ${ }^{30,43}$ The $\mathrm{Hg} 5 d_{5 / 2}$ half-widths are not easy to reconcile with cluster formation at the most dilute coverages. With such strong chemisorption occurring, even at dilute coverages, ${ }^{36}$ mercury adsorption is likely to result in $\mathrm{Hg}$ chemisorbing initially into the strong chemisorption sites first. While the growth mode for subsequent $\mathrm{Hg}$ adsorption is not known, the strong chemisorption of $\mathrm{Hg}$ at submonolayer coverages should result in $\mathrm{Hg}$ electronic structure that is substantially different for $\mathbf{H g}$ on $\mathrm{Cu}(100)$ as compared to $\mathrm{Hg}$ on $\mathrm{Si}(111)$.

A consequence of the different electronic structures for $\mathrm{Hg}$ on $\mathrm{Si}(111)$ as opposed to $\mathrm{Hg}$ on $\mathrm{Cu}(100)$ should result in a substantially different metallicity. The unique electronic structure of $\mathrm{Hg}$ (and other group-II metals) results in a surprisingly nonmetallic behavior for $\mathrm{Hg}$ monolayers on $\mathrm{Cu}(100){ }^{1}$ Destroying this unique electronic configuration for $\mathrm{Hg}$ should result in a more metallic chemisorbed $\mathrm{Hg}$ atom, even when screening from the substrate is reduced.

$\mathrm{Hg}$ on $\mathrm{Cu}(100)$ at submonolayer coverages (as seen in Fig. 4) (Ref. 1) or Ag on GaAs(110) (Ref. 28) exhibits small $5 d$ photoemission full widths at half maximum consistent with nonmetallic character. The $\mathrm{Hg} 5 d_{5 / 2}$ feature for $\mathrm{Hg}$ on $\mathrm{Si}(111)$ at $110 \mathrm{~K}$ is always observed to have a half-width of $1.05 \mathrm{eV}$ or more because of compound formation (at dilute coverage) or metallic bonding with adjacent $\mathrm{Hg}$ atoms (at high coverages). While this does not conclusively demonstrate that $\mathrm{Hg}$ is metallic at low coverages on $\mathrm{Si}(111)$ at $110 \mathrm{~K}$, it is consistent with an assignment of metallic character to the $\mathrm{Hg}$ at dilute coverages.

The quenching of the surface photovoltage has been observed $^{17,18,22,24-28}$ with metallic overlayer formation on semiconductor substrates. This quenching of the surface photovoltage is a consequence of surface conductivity and increased screening of transient charging by the metal overlayer. This is particularly true at the lower temperatures. ${ }^{17,20,21,26,27}$ The rapid quenching of the surface photovoltage with $\mathrm{Hg}$ adsorption, as seen in Fig. 3, is thus indicative of the metallic character of the mercury chemisorbed layer and, in general, of the mercury overlayer.

The surface-to-bulk core-level and chemical shifts can account for all of the Si $2 p$ binding-energy shift observed with increasing $\mathrm{Hg}$ coverage on $\mathrm{Si}(111)$ at $110 \mathrm{~K} .{ }^{36}$ This suggests that the overall effect from the band bending and photovoltage effect (when combined) is very small $(\leq 0.1$ $\mathrm{eV})$. This is consistent with the accepted principle $\mathrm{e}^{20-22}$ that the two effects result in core-level shifts in opposite directions, and band bending is diminished, effectively, at low temperatures as the photovoltage is enhanced.

The behavior of the surface photovoltage at the most dilute $\mathrm{Hg}$ coverages resembles that of a $p$-type semicon- 
ductor, not an $n$-type semiconductor. ${ }^{19,22,25,28}$ It is clear that either our surface preparation procedure for clean $\mathrm{Si}(111)$ or that mercury adsorption (and dilute $\mathrm{Hg}$ coverages left on the surface from the previous depositions) results in rapid doping of the surface to $p$ type. Using photoemission spectra to observe the addition of gap states $^{6,28}$ is therefore not likely to be a reliable indicator of a metal-nonmetal transition with increasing $\mathrm{Hg}$ coverage.

Further evidence for a metallic mercury overlayer, in the photoemission final state, may be obtained from resonant photoemission (seen in Fig. 5). Recent experimental studies of divalent metal overlayers have led to the postulate $e^{1,5,37}$ that changes in the density of conduction electrons should influence the core exciton or photohole. The screening of the hole should result in changes of the photoemission partial cross sections of the various initial states. For resonant photoemission processes increased screening leads to a decrease in the resonant photoemission intensities, even when the concentration of the characteristic element of the resonance is increased.

As noted in detail elsewhere, ${ }^{1,37-39}$ the broad feature in the CIS photoemission partial cross section is due to $5 d \rightarrow \epsilon f$ excitation, with the $5 d \rightarrow \epsilon p$ cross-section contribution decreasing from the threshold. Both the variation in the partial cross section in the photon-energy range of 40-70 eV photon energy and the peak position due to the $5 d \rightarrow \epsilon f$ excitation provide an indication of the metallic character of the $\mathrm{Hg}$ film. ${ }^{1,37-39}$ The greater the photon energy (in general) of the $5 d \rightarrow \epsilon f$ excitation maximum, the more metallic the mercury overlayer in the photoemission final state, though lattice constant and local point-group symmetry will also dramatically effect this value. Consistent with this observation, the intensity and peak position $4 d \rightarrow \epsilon f$ shape resonance of antimony clusters are profoundly influenced by the size and molecular structure of the $\mathrm{Sb}$ cluster. ${ }^{51}$ From a comparison with values obtained for various mercury overlayers, it is clear that the CIS spectra obtained for $\mathrm{Hg}$ on $\mathrm{Si}(111)$ at $110 \mathrm{~K}$ are characteristic of a metallic overlayer.

The overwhelming evidence supporting the postulate that even dilute coverages of $\mathrm{Hg}$ on $\mathrm{Si}(111)$ at $110 \mathrm{~K}$ are metallic in character in the photoemission final state appears to contradict the observation that mercury (and barium) are nonmetallic at submonolayer coverages on some metallic substrate, e.g. W(100) (Ref. 31) and $\mathrm{Cu}(100) .{ }^{1}$ It is important to realize that, while silicon is a semiconductor and is unlikely to screen adsorbed $\mathrm{Hg}$ well in the photoemission final state, at dilute coverages, $\mathrm{Hg}$ strongly chemisorbs to $\mathrm{Si}(111)$ at $110 \mathrm{~K}$. This strongly chemisorbed species cannot retain the unique property of the divalent metals such as $\mathrm{Hg}$ : a large energy gap between the fully occupied $6 s$ level and the fully unoccupied $6 p$ levels. Possibly because of depletion of the $6 s$-level occupancy, the $\mathrm{Hg}$ behaves somewhat more like an alkali metal or transition metal than adopting the necessary electronic structure ${ }^{52}$ to remain nonmetallic on a metal substrate.

If we assume a unitary sticking coefficient for $\mathrm{Hg}$ on both $\mathrm{Cu}(100)$ and $\mathrm{Si}(111)$ at $110 \mathrm{~K}$, the $\mathrm{Hg}$ overlayer coverage can be estimated., ${ }^{1,53}$ Based upon these assump- tions, $\mathrm{Hg}$ is found to be metallic at a coverage of less than 0.6 of a $\sqrt{3} \times \sqrt{3}$ monolayer $[2 \mathrm{~L}$, so that at $3-5 \mathrm{~L}$, a $\sqrt{3} \times \sqrt{3}$ monolayer is complete on $\mathrm{Si}(111)$ (Ref. 36)] by both surface photovoltage and resonant photoemission. This is consistent with the observation of a nonmetal-tometal transition at submonolayer coverages for alkalimetal adsorption on $\mathrm{Si}(100) .^{2-4}$ This is dramatically different behavior than is observed for the alkali metals on $\mathrm{GaAs}^{54-56}$ and the square lattice overlayers of $\mathrm{Hg}$ on $\mathrm{Cu}(100),{ }^{1}$ which only exhibit metallic behavior with overlayer coverages greater than one monolayer (ML). The transition for mercury occurs at a slightly smaller coverage than is observed for barium on $\mathrm{Si}(100)$ based upon the second-harmonic-generation signal. ${ }^{57}$

Even allowing for some island growth, with $\mathrm{Hg}$ adsorption on $\mathrm{Si}(111)$ at $100 \mathrm{~K}$, there is no question that $\mathrm{Hg}$ on $\mathrm{Si}(111)$ exhibits metallicity at dilute coverages and with substantially smaller coverages than that which would allow metallic-sized cluster formation. Any amount of island growth at dilute coverages would suggest that $\mathrm{Hg}$ becomes metallic at coverages less than the percolation limit of $0.5 .^{58}$

A theoretical framework of screening can assist in our understanding of the dramatic changes in apparent metallic character in the photoemission final state for various $\mathrm{Hg}$ overlayers. Simple theoretical models support the postulate that resonant photoemission intensities become weaker with increasing electron itinerancy. For two-dimensional systems the screening effect is much weaker than for three-dimensional systems, consistent with recent experiments. ${ }^{1,5}$ Resonant photoemission intensities may therefore not only provide an indication of screening in the final state, but the initial state as well. For ultrathin metal overlayers (1-2 ML) and surfaces, resonant photoemission will be more sensitive to changes in electron screening lengths than will be the case for thicker films.

In the metal-nonmetal transition of the Mott type, the nonmetal is an excitonic insulator ${ }^{59-61}$ and the excitons unbind at the metal-nonmetal transition. ${ }^{61}$ Free carriers (itinerant electrons) will affect the formation of excitons by either screening the Coulomb field between the electron-hole pair or shorten the lifetime of the exciton. ${ }^{62}$ The core exciton is believed ${ }^{1}$ to be similarly affected.

Models to describe screening in two dimensions (in the $x-y$ plane) have been outlined. ${ }^{63-65}$ For a pure twodimensional free-electron system, the relationship between the density of electrons and Fermi momentum is

$$
k_{F}=\left(\frac{2 \pi n_{s}}{g_{v}}\right)^{1 / 2},
$$

where $g_{v}=d E / d k$.

When there exists an external charge source with density $\rho_{\text {ext }}$, this source will induce a new distribution of electrons in the system and also a local potential. The screening effect to such an external source by the electrons can be estimated by using Poisson's equation. Applying Thomas-Fermi screening theory, ${ }^{63}$ a relationship between the local potential $\phi(r)$ and the screening parameter $l_{S}$ can be obtained from 


$$
\nabla(\bar{\kappa} \nabla \phi)-2 \bar{\kappa} l_{S} \phi=-4 \pi \bar{\rho}_{\text {ext }},
$$

where $\bar{\kappa}$ is the average dielectric constant of the system (including the external charge) and $\bar{\rho}_{\text {ext }}=\left.\rho_{\text {ext }}\right|_{z=0}$ is the charge density in the two-dimensional system induced by $\rho_{\text {ext }}$. The screening parameter $l_{S}$ is

$$
l_{S}=\frac{2 \pi e^{2}}{\bar{\kappa}} \frac{d n_{s}}{d E_{F}} .
$$

According to the free-electron-gas approximation at finite temperature, $l_{S}$ can be explicitly written as ${ }^{64-68}$

$$
l_{S}=\frac{2}{a}\left[1-\exp \left(\pi \hbar^{2} n_{S} / m^{*} k_{B} T\right)\right],
$$

where $a=\bar{\kappa} \hbar^{2} / m^{*} e^{2}$ ( $m^{*}$ is the effective mass of electron). In the high-temperature approximation, we have

$$
\bar{\kappa} l_{S}=\frac{2 \pi e^{2} n_{S}}{k_{B}} T .
$$

Thus screening increases with increased density of states at the Fermi energy (increasing conduction electrons). This appears to be valid for both the pure twodimensional systems (monolayer films) and some multilayer film thicknesses. ${ }^{63}$ For metal monolayers such as mercury, $n_{S}$ is seen to increase greatly with decreasing lattice constant under some conditions ${ }^{1}$ and is shown in Fig. 6. This change in $n_{S}$ does appear to result in changes in the photoemission partial cross sections that reflect an increase in the screening length $l_{S}$ (Ref. 1) and are summarized in Table $I$. The correlation is not simple, however, due to the presence of Hubbard bands or Mott gap states. ${ }^{1}$

For a hole of charge $q$ at $r=0$ and $z_{0} \leq 0$, a solution to the potential $\phi(r)$ may be written, ${ }^{64}$ in the asymptotic behavior for $l_{S} r \gg 1$, as

$$
\phi(r) \sim \frac{q\left(1+l_{S} z_{0}\right)}{\bar{\kappa} l_{S}^{2} r^{3}} .
$$

For two dimensions the screening effect to a hole is therefore much weaker than for three dimensions, where the effective potential is

$$
\phi(r) \sim \frac{1}{r} \exp \left(-l_{S} r\right) .
$$

Similar results have also been obtained earlier by Stern and Howard ${ }^{65}$ for thin films (not a pure two-dimensional system). For core excitons the result will be qualitatively similar. A comparison of two dimension with three dimensions indicates that the screening is far better in three dimensions, indicating that longer core-exciton lifetimes exist in two dimensions if all other parameters are equal.

In the three-dimensional expanded mercury system, the Mott excitonic insulating phase can be obtained only if the lattice constant $a$ is larger than 4-5 $\AA$ (actually, 5.1-5.9 $\AA$ for fcc mercury and 4.2-5.0 $\AA$ for bcc mercury). ${ }^{59}$ In the two-dimensional case, the corresponding insulation phase can be reached even if the lattice constant is just about $3 \AA$ (Ref. 69) [actually $2.9 \AA$ for $\mathrm{Hg}$ on $\mathrm{Ni}(111),{ }^{35}$ and $3.16 \AA$ for $\mathrm{Hg} / \mathrm{W}(100),{ }^{31}$ and $3.22 \AA$ for $\mathrm{Hg} / \mathrm{Cu}(100)$ (Ref. 1)]. This means that, to reach the excitonic insulating phase (i.e., to get enough Coulomb interaction between holes and electrons so that the electrons are localized), a larger mercury lattice constant is required in the three-dimensional case than in the twodimensional case. This is necessary to compensate the stronger screening effect in three dimension than in two dimensions, since $l_{S}$ is proportional to $n_{S}$, which is proportional to the reciprocal of the lattice constant (i.e., $1 / a)$.

Alternatively, considering an effective Hamiltonian of an electron-hole pair,

$$
H=\frac{p^{2}}{2 \mu}+V_{S}(r),
$$

where $\mu$ is the effective reduced mass, then the screened Coulomb potential for the pure two-dimensional system can be written, ${ }^{65,67}$ using a Fourier-Bessel expansion method to solve Eq. (2) [Eq. (5) is the asymptotic behavior of the following solution], as

$$
V_{S}(r)=\left(\frac{-q e}{\kappa}\right)\left(\frac{1}{r}-l_{S} \int_{0}^{\infty} \frac{J_{0}(l r)}{l+l_{S}} d l\right),
$$

which indicates that screening is much weaker in the two-dimensional case than in the three-dimensional case. Using a trial exciton wave function as

$$
\psi(r)=N \exp (-\beta r / 2),
$$

TABLE I. Comparison of the CIS-derived $5 d \rightarrow \epsilon f$ excitation photoemission partial cross-section maximum for various $\mathrm{Hg}$ overlayers. Film thicknesses are in nominal monolayers, while the photon energies are in units of $\mathrm{eV}$.

\begin{tabular}{lccccc}
\hline \hline & $\begin{array}{c}\text { Hg film } \\
\text { thickness } \\
\text { Substrate }\end{array}$ & Symmetry & $\begin{array}{c}5 d \rightarrow \epsilon f \\
\text { maximum } \\
(\mathrm{eV})\end{array}$ & Character & Reference \\
\hline $\mathrm{Si}(111)$ & 1 & $C_{6 v}$ & 59 & metallic & this work \\
$\mathrm{Si}(111)$ & 10 & $C_{4 v}$ & 59 & metallic & this work \\
$\mathrm{Ag}(100)$ & 2 & $C_{2 v}$ & 58 & metallic & this work \\
$\mathrm{Ag}(100)$ & 5 & disordered & $<45$ & metallic & $37-39$ \\
$\mathrm{Ag}(100)$ & 5 & $C_{4 v}$ & 55 & nonmetallic & 38 \\
$\mathrm{Cu}(100)$ & $<1$ & $C_{4 v}$ & $59-60$ & metallic & 1 \\
$\mathrm{Cu}(100)$ & $>1$ & & $36-38$ & nonmetallic & $40-42$ \\
$\mathrm{Gas}$ phase & & & & & \\
\hline \hline
\end{tabular}




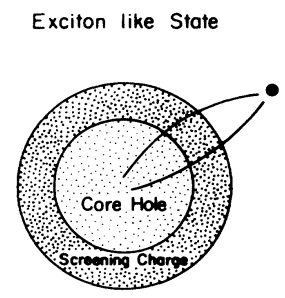

Localization $\rightarrow$ Poor Screening

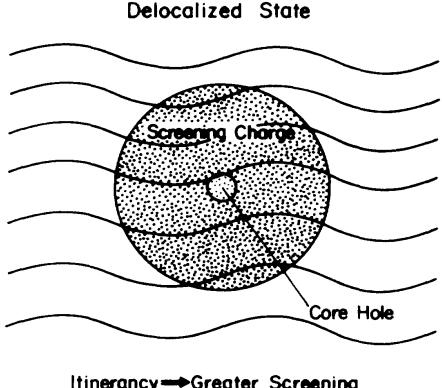

Itinerancy $\rightarrow$ Greater Screening

FIG. 7. Schematic diagram of the influence of strong and weak screening upon a core exciton in two dimensions.

where $\beta$ is the variational parameter and $N=\beta(2 \pi)^{1 / 2}$. As the wave function becomes more extended (i.e., $\beta^{2}$ decreases) as $l_{S}$ increases, as schematically shown in Fig. 7. The binding energy of the exciton can be obtained by minimizing the expectation value of the energy of the exciton $E=(\psi, H \psi) /(\psi, \psi)$. One can find out that the exciton binding energy decreases as the screening parameter increases.

Since the optical-absorption strength at the exciton peak is proportional to $|\psi(0)|^{2}$ or $\beta^{2}$, which decreases as $l_{S}$ increases, ${ }^{65}$ the intensity at the peak will decrease as the screening parameter increases. Such a change reduces the resonant photoemission intensity as a result of a decrease in the core-exciton lifetime. ${ }^{1,5}$ The qualitative decrease in resonant photoemission intensities upon increasing the dimensionality, as is expected from Eq. (6) as compared with (7) or Eq. (9) as compared with the screened Coulomb potential energy in three dimensions, is seen experimentally. ${ }^{1,5}$ In the $5 d \rightarrow \epsilon f$ excitation, the outgoing photoelectron interacts with a screened photohole. Increased screening shifts the cross-section maximum to higher photoenergies (shorter wavelengths), as summarized in Table $I$, and diminishes the variation in cross section, as seen in Fig. 5 and reported in detail previously. ${ }^{1}$

A structural phase transition for mercury on $\mathrm{Ni}(111)$ has been observed by Singh and Jones. ${ }^{35}$ A comparison of the structural data of Singh and Jones with theory ${ }^{69}$ suggests that the structural phase transition permits the density of electrons near $E_{F}$ to be maximized (i.e., $n_{S}$ is maximized). ${ }^{35}$ In view of these results, screening and initial-state hybridization appears to be maximized to lower the free energy of the initial state and is confirmed by recent studies of the energetics of mercury overlayers. ${ }^{43}$ Changes to both $N_{E f}$ and electron itinerancy will affect the screening parameter $l_{S}$, and electron localization $^{1}$ must therefore be included in a more detailed

model.

For mercury overlayers on metal surfaces, a gradual change in $n_{S}$ is observed with changing lattice constant. The results are in relatively good agreement ${ }^{1}$ with calculated values obtained for a free-standing $\mathrm{Hg}$ monolayer ${ }^{69}$ consistent with the postulate that $\mathrm{Hg}$ only weakly interacts with the $\mathrm{W}(100), \mathrm{Cu}(100), \quad \mathrm{Ag}(100)$, and $\mathrm{Cu}_{3} \mathrm{Au}(100)$ surfaces unlike $\mathrm{Hg}$ on $\mathrm{Si}(111)$, as summarized in Fig. 6.

Other models for screening and delocalization effects which accompany metal deposition on semiconductor surfaces ${ }^{70}$ suggest that the presence of defects and impurity levels affect the Fermi-level position. Given the number of ambiguities that exist with regard to the interface electronic and morphologic structure for $\mathbf{H g}$ on $\mathrm{Si}(111)$, a more detailed description of screening effects has not been attempted.

\section{CONCLUSION}

Because of strong chemisorption, at submonolayer coverages, the mercury overlayer exhibits more metallic character on $\mathrm{Si}(111)$ than on $\mathrm{Cu}(100)$. With metallic overlayer formation, surface photovoltage band shifts are reduced with $\mathrm{Hg}$ adsorption on $\mathrm{Si}(111)$ at $100 \mathrm{~K}$. This quenching of the surface photovoltage is a consequence of surface conductivity and increased screening by the metallic overlayer.

We find that with weak chemisorption, as is observed with $\mathrm{Hg}$ on $\mathrm{Cu}(100),{ }^{43} \mathrm{Hg}$ exhibits substantial nonmetallic behavior in the photoemission final state, ${ }^{1}$ despite increased screening from the substrate. We speculate that this absence of nonmetallic behavior for $\mathrm{Hg}$ on $\mathrm{Si}(111)$ at dilute coverages may be attributable to modification of the unique electronic structure of the group-II metals, ${ }^{52}$ despite decreased screening from the substrate. Island formation, substantial enough to form $\mathrm{Hg}$ clusters sufficiently large so as to be metallic, cannot be reconciled with our data at dilute coverages.

The simple models of screening length in two dimensions, while not including substrate contributions, do provide a qualitative description of resonant photoemission results obtained thus far. ${ }^{1,5}$

\section{ACKNOWLEDGMENTS}

The authors acknowledge helpful conversations with A. Kahn and L. Brillson. The authors would also like to thank G. K. Wertheim and S. D. Kevan for sharing the results of their work prior to publication. This work was funded by the NSF (DMR-88-20779). The photoemission studies were carried out at the Synchrotron Radiation Center in Stoughton, WI, which is funded by the NSF.
*To whom all correspondence should be addressed.

${ }^{1}$ P. A. Dowben, D. LaGraffe, Dongqi Li, G. Vidali, L. Zhang, L. Dottl, and M. Onelion, Phys. Rev. B 43, 10677 (1991).

${ }^{2}$ H. Tochihara, Surf. Sci. 126, 523 (1983).

${ }^{3}$ S. Arekat, G. L. Richmond, and S. D. Kevan, J. Vac. Sci. Technol. A (to be published); S. Arekat, S. D. Kevan, and G.
Richmond (unpublished).

${ }^{4}$ D. M. Riffe, G. K. Wertheim, J. E. Rowe, and P. H. Citrin, Phys. Rev. B 45, 3532 (1992).

${ }^{5}$ P. A. Dowben and D. LaGraffe, Phys. Lett. A 144, 193 (1990).

${ }^{6}$ C. Calandra, O. Bisi, and G. Ottaviani, Surf. Sci. Rep. 4, 271

(1985), and references therein. 
${ }^{7}$ J. H. Weaver, Zhangda Lin, and F. Xu, in Surface Segregation Phenomena, edited by P. A. Dowben and A. Miller (CRC, Boca Raton, 1990), p. 259; Z. Lin, F. Xu, and J. H. Weaver, Phys. Rev. B 36, 5777 (1987).

${ }^{8}$ E. Daugy, J. M. Layet, P. Matheiz, and F. Salvan, J. Vac. Sci. Technol. B 1, 546 (1983).

${ }^{9}$ D. D. Chambliss, T. N. Rhodin, J. E. Rowe, and H. Shigekawa, J. Vac. Sci. Technol. A 7, 2449 (1989).

${ }^{10}$ T. Kinoshita, Y. Enta, H. Ohta, Y. Yaegashi, S. Suzuki, and S. Kono, Surf. Sci. 204, 405 (1988); T. Kinoshita, S. Kono, and H. Nagayoshi, J. Phys. Soc. Jpn. 56, 2511 (1987).

${ }^{11}$ G. Margaritondo, J. E. Rowe, and S. B. Christman, Phys. Rev. B 14, 5396 (1976).

${ }^{12}$ R. I. G. Uhrberg and G. V. Hansson, Crit. Rev. Solid State Mater. Sci. 17, 133 (1991). A. L. Wachs, A. P. Shapiro, T. C. Hsieh, and T.-C. Chiang, Phys. Rev. B 33, 1460 (1986).

${ }^{13}$ D. D. Chambliss, T. N. Rhodin, and R. V. Kasowski, J. Vac. Sci. Technol. A 6, 1499 (1988).

${ }^{14}$ P. A. Dowben, D. LaGraffe, and M. Onellion, J. Phys. Condens. Matter 1, 6571 (1989).

${ }^{15}$ F. Keith Perkins, R. A. Rosenberg, SunWoo Lee, and P. A. Dowben, J. Appl. Phys. 69, 4103 (1991).

${ }^{16}$ A. L. Wachs, T. Miller, T. C. Hsieh, A. P. Shapiro, and T. C. Chiang, Phys. Rev. B 32, 2326 (1985); L. Ley, M. Cardona, and R. A. Pollak, in Photoemission in Solids II, Topics in Applied Physics Vol. 47 (Springer, New York, 1979), pp. 11 -172; D. Haneman, Rep. Prog. Phys. 50, 1045 (1987), and references therein.

${ }^{17}$ M. Alonso, R. Cimino, and K. Horn, J. Vac. Sci. Technol. A 9, 891 (1991); M. Alonso, R. Cimino, and K. Horn, Phys. Rev. Lett. 64, 1947 (1990); K. Horn, M. Alonso, and R. Cimino, Appl. Surf. Sci (to be published).

${ }^{18}$ C. M. Aldao, G. D Waddill, P. J. Benning, C. Capasso, and J. H. Weaver, ibid. 41, 6092 (1990); C. M. Aldao, I. M. Vitomirov, G. D. Waddill, S. G. Anderson, and J. H. Weaver, ibid. 41, 2800 (1990); G. D Waddill, Tadahiro Komeda, Y.-N. Yang, and J. H. Weaver, ibid. 41, 10283 (1990).

${ }^{19}$ J. E. Demuth, W. J. Thompson, N. J. DiNardo, and R. Imbihl, Phys. Rev. Lett. 56, 1408 (1986).

${ }^{20}$ M. H. Hecht, Phys. Rev. B 4, 7918 (1990).

${ }^{21}$ M. H. Hecht, J. Vac. Sci. Technol. B 8, 1018 (1990).

${ }^{22}$ S. Chang, I. M. Vitomirov, L. J. Brillson, D. F. Rioux, P. D. Kirchner, G. D. Pettit, J. M. Woodall, and M. H. Hecht, Phys. Rev. B 41, 12299 (1990); S. Chang, I. M. Vitomirov, L. J. Brillson, D. F. Rioux, P. D. Kirchner, G. D. Pettit, and J. M. Woodall, J. Vac. Sci. Technol. B 9, 2129 (1991).

${ }^{23}$ R. J. Hamers and K. Markert, J. Vac. Sci. Technol. A 8, 3524 (1990).

${ }^{24}$ R. Cao, M. Miyano, T. Kendelewicz, K. K. Chin, Ingolf Landau, and W. E. Spicer, J. Vac. Sci. Technol. B 5, 998 (1987).

${ }^{25}$ K. Stiles, A. Kahn, D. G. Kilday, and G. Margaritondo, J. Vac. Sci. Technol. B 5, 987 (1985).

${ }^{26}$ D. Mao, A. Kahn, M. Marsi, and G. Margaritondo, Phys. Rev. B 42, 3228 (1990)

${ }^{27}$ D. Mao, A. Kahn, M. Marsi, and G. Margaritondo, J. Vac. Sci. Technol. A 9, 898 (1991); D. Mao, A. Kahn, G. LeLay, M. Marsi, Y. Hwu, G. Margaritondo, M. Santos, M. Shayegan, L. T. Florez, and J. P. Harbison, J. Vac. Sci. Technol. B 9, 2083 (1991).

${ }^{28}$ K. Stiles and A. Kahn, Phys. Rev. Lett. 60, 440 (1988).

${ }^{29}$ M. Onellion, Y. J. Kime, P. A. Dowben, and N. Tache, J. Phys. C 20, L633; P. A. Dowben, S. Varma, Y. J. Kime, D. R. Mueller, and M. Onellion, Z. Phys. B 73, 247 (1988).
${ }^{30}$ P. A. Dowben, Y. J. Kime, S. Varma, M. Onellion, and J. L. Erskine, Phys. Rev. B 36, 2519 (1987); P. A. Dowben, M. Onellion, and Y. J. Kime, Scanning Microsc. 2, 177 (1988); M. Onellion, J. L. Erskine, Y. J. Kime, S. Varma, and P. A. Dowben, Phys. Rev. B 33, 8833 (1968).

${ }^{31}$ W. F. Egelhoff, D. L. Perry, and J. W. Linnett, Surf. Sci. 54, 670 (1976).

${ }^{32}$ G. E. Becker and H. G. Hagstrum, J. Vac. Sci. Technol. 10, 31 (1973).

${ }^{33}$ S. Svensson, N. Martensson, E. Basilier, P. A. Malquist, U. Gelius, and K. Siegbahn, J. Electron Spectrosc. Relat. Phenom. 9, 51 (1976).

${ }^{34}$ P. A. Dowben, Y. J. Kime, D. LaGraffe, and M. Onellion, Surf. Interface Anal. 15, 163 (1990).

${ }^{35}$ N. K. Singh and R. G. Jones, Chem. Phys. Lett. 155, 463 (1988); Surf. Sci. 232, 243 (1990).

${ }^{36}$ Dongqi Li, Jingsu Lin, Wei Li, Sunwoo Lee, G. Vidali, and P. A. Dowben (unpublished).

${ }^{37}$ S. Varma, Y. J. Kime, P. A. Dowben, M. Onellion, and J. L. Erskine, Phys. Lett. A 116, 66 (1986).

${ }^{38}$ P. A. Dowben, M. Onellion, S. Varma, Y. J. Kime, and J. L. Erskine, J. Vac. Sci. Technol. A 7, 2070 (1989); M. Onellion, P. A. Dowben, and J. L. Erskine, Phys. Lett. A 130, 171 (1988).

${ }^{39}$ S. Varma, Y. J. Kime, D. LaGraffe, P. A. Dowben, M. Onellion, and J. L. Erskine, J. Chem. Phys. 93, 2819 (1990).

${ }^{40}$ J. L. Dehnmer and J. Berkowitz, Phys. Rev. A 6, 484 (1974).

${ }^{41}$ F. Keller and F. Combet-Famoux, J. Phys. B 12, 2821 (1979).

${ }^{42}$ W. R. Johnson, V. Radojevic, Pranawa Deshmukh, and K. T. Cheng, Phys. Rev. A 25, 337 (1982).

${ }^{43}$ Y. J. Kime, Jiandi Zhang, and P. A. Dowben, Surf. Sci. (to be published).

${ }^{44} Y$. J. Kime, Jiandi Zhang, and P. A. Dowben, in Structure/Property Relationships for Metal/Metal Interfaces, edited by A. D. Romig, D. E. Fowler, and P. D. Bristowe, MRS Symposia Proceedings No. 229 (Materials Research Society, Pittsburgh, 1991), p. 221.

${ }^{45}$ R. G. Jones and A. W.-L. Tong, Surf. Sci. 188, 87 (1987).

${ }^{46}$ N. K. Singh and R. G. Jones, Surf. Sci. 232, 229 (1990).

${ }^{47}$ R. G. Jones and D. L. Perry, Surf. Sci. 71, 59 (1978); 82, 540 (1979).

${ }^{48}$ R. G. Jones and D. L. Perry, Vacuum 31, 493 (1981).

${ }^{49}$ Kenjiro Oura, Koji Sumitomo, Tadashi Kobayashi, Toshihiro Kinoshita, Yasunori Tonaka, Fumiya Shoji, and Itsuo Katayama, Surf. Sci. 254, L460 (1991); E. L. Bullock, G. S. Herman, M. Yamada, D. J. Friedman, and C. S. Fadley, Phys. Rev. B 41, 1703 (1990); W. C. Fan, A. Ignatiev, H. Huang, and S. Y. Tong, Phys. Rev. Lett. 62, 1516 (1989); G. Rossi, I. Abbati, L. Braicovitch, I. Lindau, and W. E. Spicer, Surf. Sci. 112, L765 (1981); K. Higashima, S. Kono, and T. Sagawa, ibid. 175, L794 (1986); T. Kinoshita, S. Kono, and T. Sagawa, Phys. Rev. B 34, 3011 (1986); J. Stöhr, R. Jaeger, G. Rossi, T. Kendelewicz, and I. Lindau, Surf. Sci. 134, 813 (1983); X. Vandré, X. Incoccia, and X. Kaindl, ibid. 225, 233 (1990); T. Hiloma, H. H. Weitering, D. R. Heslinga, and T. M. Klapwijk, Appl. Surf. Sci. 48/49, 366 (1991); K. Spiegel, Surf. Sci. 7, 125 (1967); G. LeLay, M. Manneville, and R. Kern, ibid. 72, 405 (1978); F. Wehking, H. Beckermann, and R. Niedermayer, ibid. 71, 364 (1978); T. N. Doust and S. P. Tear, ibid. 251/252, 568 (1991); J. J. Lander and J. Morrison, ibid. 2, 553 (1964).

${ }^{50}$ C. Bréchignac, M. Broyer, Ph. Cahuzac, G. Delacretaz, P. Labastie, J. P. Wolf, and L. Wöste, Phys. Rev. Lett. 60, 275 
(1988); M. E. Garcia, G. M. Pastor, and K. H. Bennemann, ibid. 67, 1142 (1991); C. Bréchignac, M. Broyer, Ph. Cahuzac, G. Delacretaz, P. Labastie, and L. Wörste, Chem. Phys. Lett. 120, 559 (1985); K. Rademann, B. Kaiser, U. Even, and F. Hensel, Phys. Rev. Lett. 59, 2319 (1987); K. Rademann, B. Kaiser, T. Rech, and F. Hensel, Z. Phys. 12, 431 (1989); K. Rademann, Ber. Bunsenges. Phys. Chem. 93, 653 (1989).

${ }^{51}$ C. Bréchignac, M. Broyer, Ph. Cahuzac, M. deFrutos, P. Labastie, and J.-Ph. Roux, Phys. Rev. Lett. 67, 1222 (1991).

${ }^{52}$ A. R. Miedema and Dorleijn, Philos. Mag. B 43, 251 (1981).

${ }^{53}$ P. A. Dowben, Y. J. Kime, C. W. Hutchings, Wei Li, and G. Vidali, Surf. Sci. 230, 113 (1990); C. W. Hutchings, P. A. Dowben, Y. J. Kime, W. Li, M. Karimi, C. Moses, and G. Vidali, in Atomic Scale Structure of Interfaces, edited by R. Brigans, R. Feenstra, and M. Gibson, MRS Symposia Proceedings No. 159 (Materials Research Society, Pittsburgh, 1990), p. 133.

${ }^{54}$ N. J. DiNardo, T. Maedo Wong, and E. W. Plummer, Phys. Rev. Lett. 65, 2177 (1990).

${ }^{55}$ A. B. McLean, D. Heskett, Daixing Tang, and N. J. DiNardo, Phys. Rev. Lett. 65, 624 (1990).

${ }^{56}$ L. J. Whitman, J. A. Stroscio, R. A. Dragoset, and R. J. Celotta, Phys. Rev. Lett. 66, 1338 (1991).

${ }^{57}$ R. W. J. Hollering, H. W. L. Lindelauf, P. A. M. von der Heide, and M. P. C. Krijn, J. Vac. Sci. Technol. A 8, 3997
(1990).

${ }^{58}$ Dietrich Stauffer, Introduction to Percolation Theory (Taylor and Francis, London, 1985), p. 17.

${ }^{59}$ F. Yonezawa and T. Ogawa, Prog. Theor. Phys. Suppl. 72, 1 (1982), and references therein.

${ }^{60}$ N. F. Mott, Rev. Mod. Phys. 40, 677 (1968).

${ }^{61}$ N. F. Mott, Metal Insulator Transitions, 2nd ed. (Taylor and Francis, London, 1990).

${ }^{62}$ L. A. Turkevich and M. H. Cohen, Phys. Rev. Lett. 53, 2323 (1984).

${ }^{63}$ T. Adno, A. B. Fowler, and S. Stern, Rev. Mod. Phys. 54, 4 (1982).

${ }^{64}$ J. Lee, H. N. Spector, and P. Melman, J. Appl. Phys. 58, 1893 (1985).

${ }^{65}$ F. Stern and W. E. Howard, Phys. Rev. 163, 813 (1967); F. Stern, Phys. Rev. Lett. 18, 546 (1967).

${ }^{66}$ O. Hipolito and V. B. Campos, Phys. Rev. B 19, 599 (1981).

${ }^{67}$ P. Price, J. Vac. Sci. Technol. 19, 599 (1981).

${ }^{68}$ W. E. Edelstein and H. N. Spector, Surf. Sci. 224, 581 (1989).

${ }^{69}$ H. J. F. Jansen, A. J. Freeman, M. Weinert, and E. Wimmer, Phys. Rev. B 28, 593 (1983).

${ }^{70}$ R. Ludeke, G. Jezequel, and A. Taleb-Ibrahimi, J. Vac. Sci. Technol. B 1277, (1988); Y. Ma, J. E. Rowe, E. E. Chaban, C. T. Chen, R. L. Headrick, G. M. Meigs, S. Modesti, and F. Sette, Phys. Rev. Lett. 65, 2173 (1990). 